\title{
Growth of Sobolev norms of solutions of linear Schrödinger equations on some compact manifolds
}

\author{
J.-M. Delort \\ Université Paris 13, Institut Galilée, \\ CNRS, UMR 7539, Laboratoire Analyse Géométrie et Applications \\ 99, Avenue J.-B. Clément, \\ F-93430 Villetaneuse
}

\begin{abstract}
We give a new proof of a theorem of Bourgain [4], asserting that solutions of linear Schrödinger equations on the torus, with smooth time dependent potential, have Sobolev norms growing at most like $t^{\epsilon}$ when $t \rightarrow+\infty$, for any $\epsilon>0$. Our proof extends to Schrödinger equations on other examples of compact riemannian manifolds.
\end{abstract}

\section{Introduction}

The main goal of this paper is to give a simpler proof of a theorem of Bourgain [4], and to obtain extensions and generalizations of this result. Let $M=\mathbb{T}^{d}$ be the standard torus $\mathbb{T}^{d}=(\mathbb{R} / 2 \pi \mathbb{Z})^{d}$, and let $V: \mathbb{R} \times \mathbb{T}^{d} \rightarrow \mathbb{R}$ be a smooth function, bounded as well as its derivatives. Let $u$ be the solution of the Schrödinger equation

$$
\begin{aligned}
\left(i \partial_{t}-\Delta+V(t, x)\right) u & =0 \\
\left.u\right|_{t=0} & =u_{0}
\end{aligned}
$$

with $u_{0} \in H^{s}(M)$.

Theorem 1.1 (Bourgain) For any $s>0$, there is a constant $C>0$ such that for any $u_{0} \in$ $H^{s}\left(\mathbb{T}^{d}\right)$, the solution $u$ to (1.1) satisfies, for any $t \in \mathbb{R}$,

$$
\|u(t, \cdot)\|_{H^{s}} \leq C(1+|t|)\left\|u_{0}\right\|_{H^{s}} .
$$

Remark: Actually, we shall obtain a slightly better estimate than (1.2), namely

$$
\|u(t, \cdot)\|_{H^{s}} \leq C\left[\left\|u_{0}\right\|_{H^{s}}+|t|\left\|u_{0}\right\|_{L^{2}}\right] .
$$

This work was partially supported by the ANR project Equa-disp. 
Since $V$ is real valued, $\|u(t, \cdot)\|_{L^{2}}=\left\|u_{0}\right\|_{L^{2}}$, so that interpolating with (1.2), one obtains that for any $s \in \mathbb{R}$, any $\epsilon>0$, there is $C>0$ such that for any $u_{0} \in H^{s}\left(\mathbb{T}^{d}\right)$, any $t \in \mathbb{R}$,

$$
\|u(t, \cdot)\|_{H^{s}} \leq C(1+|t|)^{\epsilon}\left\|u_{0}\right\|_{H^{s}} .
$$

Inequality (1.3) is the statement of Proposition 1 in Bourgain [4]. If one assumes that $V$ is analytic and time quasi-periodic, it had been proved previously by Bourgain [3] that (1.3) holds with $(1+|t|)^{\epsilon}$ replaced by some power of $\log t$ when $t \geq 2$. In dimension $d=1$, Wei-Min Wang [13] showed recently that such a logarithmic bound holds for any real analytic potential, whose holomorphic extension to a complex strip around $\mathbb{R} \times \mathbb{S}^{1}$ is bounded, adapting the method of [4] to this setting. Let us mention that Bourgain [3] constructed examples of time periodic potentials for which $\|u(t, \cdot)\|_{H^{s}}$ is bounded from below by a power of $\log t$ when $s>0$ and $t \rightarrow+\infty$. On the other hand, in a recent work, Eliasson and Kuksin [11] have shown that if the potential $V$ on $\mathbb{R} \times \mathbb{T}^{d}$ is analytic in space, quasi-periodic in time, and small enough, then for most values of the parameter of quasi-periodicity, the equation reduces to an autonomous one. Consequently, the Sobolev norm of the solution is uniformly bounded. For Schrödinger equations on the circle, Wei-Min Wang [14] has constructed an explicit example of a small time periodic potential for which the solutions of the corresponding equation have bounded Sobolev norms.

The proof of Theorem 1.1 given by Bourgain in [4] (see also appendix 1 of [5] for the case $d=1$ ) relies on reduction to a time periodic problem and then uses space-time Fourier series decompositions. The alternative proof ${ }^{1}$ we propose here makes use only of "symbolic calculus" properties, and is stationary in essence: we construct for every fixed time an operator, which conjugates equation (1.1) to a similar equation, where the potential $V$ has been replaced by another operator $V^{\prime}$, exactly commuting to a modified Laplacian. The conclusion then follows from energy inequalities and characterization of Sobolev regularity in terms of that modified Laplacian.

In section 2 below, we shall actually state and prove an abstract version of Theorem 1.1, where $-\Delta, V$ are replaced by operators assumed to satisfy a list of assumptions. We check in section 3 that these assumptions hold for the Laplacian on $\mathbb{T}^{d}$. We use for that the same geometric properties of the spectrum of $-\Delta$ on $\mathbb{T}^{d}$ as Bourgain in [4].

A natural question is to know whether a result like Theorem 1.1 holds on other compact manifolds than $\mathbb{T}^{d}$. We are able to check that our abstract result does apply to a couple of classes of compact manifolds.

Recall that a Zoll manifold is a compact manifold whose geodesic flow is periodic. The sphere is such a manifold, and we refer to the book of Besse [2] for other examples. Our second result is the following one:

Theorem 1.2 Let $M$ be a Zoll manifold, and let $V: M \times \mathbb{R} \rightarrow \mathbb{R}$ be smooth, with all its derivatives bounded. For any $s>0$, there is a constant $C>0$ such that, for any $u_{0} \in H^{s}(M)$,

\footnotetext{
${ }^{1}$ After acceptation of this paper, we have been informed by Jean-Michel Combes that related results have been published by Barbaroux and Joye in [1].
} 
the solution $u$ of (1.1) satisfies, for any $t \in \mathbb{R}$,

$$
\|u(t, \cdot)\|_{H^{s}} \leq C(1+|t|)\left\|u_{0}\right\|_{H^{s}} .
$$

Again, one deduces from (1.4) an estimate of the form (1.3). The proof of Theorem 1.2 is given in section 4 . We check that the assumptions of our abstract result of section 2 hold for $-\Delta$ on a Zoll manifold. The key point is a separation property of the eigenvalues of $-\Delta$ on $M$, which extends to higher dimensions the fact that on $\mathbb{S}^{1}$ these eigenvalues are the squares of the integers.

Theorems 1.1 and 1.2 above apply to examples of manifolds which are not stable under any reasonable perturbation. One may ask if it is possible to get an estimate of the type of (1.2) for solutions of Schrödinger equations, with time dependent potential on a continuous family of compact manifolds. This is the aim of our third theorem, where we study the case of some surfaces of revolution, for which the spectrum of the Laplace operator may be precisely described by results of Colin de Verdière $[8,9]$.

Consider on the sphere $\mathbb{S}^{2}$ a metric of revolution $g$. Denote by $L$ the length of a meridian geodesic going from the North pole to the South pole, and by $\sigma$ the arc-length along that geodesic. Assume that outside the poles $g$ may be written $g=a(\sigma) d \theta^{2}+d \sigma^{2}$, where $\theta$ is the polar angle, $a:[0, L] \rightarrow \mathbb{R}_{+}$is a smooth function satisfying $a(0)=a(L)=0, a(\sigma)>0$ if $\sigma \in] 0, L\left[\right.$, having a unique critical point $\sigma_{0}$ in $] 0, L[$, and suppose that this critical point is nondegenerate (so that $a^{\prime \prime}\left(\sigma_{0}\right)<0$ ). We refer to page 63 of [9] for pictures of surfaces that do or do not satisfy the above condition. Intuitively, it means that if $M$ is isometrically embedded in $\mathbb{R}^{3}$, the distance from the axis of rotation to the point on a meridian geodesic with parameter $\sigma$ (which is given by $\sqrt{a(\sigma)}$ ) has only one extremum. In other words, $M$ has only one equator. Note also that the assumptions are stable under small perturbations of $a$.

Theorem 1.3 Let $M$ be the above surface, $V: \mathbb{R} \times M \rightarrow \mathbb{R}$ a smooth potential, bounded as well as all its derivatives. Then estimate (1.2) holds for solutions of (1.1) with Cauchy data $u_{0} \in H^{s}(M)$.

Again, the apparently stronger estimate (1.3) holds as well.

The proof of Theorem 1.3 will be given in section 5 . We shall check that the assumptions of our abstract theorem hold true using the results of Colin de Verdière $[8,9]$, which allow one to describe the (joint) spectrum of the Laplacian on the surface we consider from the image of a subset of the lattice $\mathbb{Z}^{2}$ by a symbol.

The abstract result of section 2 does not apply just to Schrödinger operators. In section 6 we treat the example of a "linear KdV" equation, proving the following:

Theorem 1.4 Let $W: \mathbb{R} \times \mathbb{S}^{1} \rightarrow \mathbb{R}$ be a smooth function of $(t, x)$ bounded as well as its derivatives. Consider the equation on $\mathbb{R} \times \mathbb{S}^{1}$

$$
\begin{aligned}
\partial_{t} u+\partial_{x}^{3} u+W \partial_{x} u+\partial_{x}(W u) & =0 \\
\left.u\right|_{t=0} & =u_{0} .
\end{aligned}
$$


Then for any $s>0$, there is a constant $C>0$ such that, for any $u_{0} \in H^{s}\left(\mathbb{S}^{1}\right)$, the solution of (1.5) satisfies (1.2) for any $t \in \mathbb{R}$.

Using again conservation of the $L^{2}$ norm of $u$, one deduces from an estimate of type (1.2) a bound of the form of (1.3).

\section{Abstract result}

This section is devoted to the statement and proof of a general result from which theorems 1.1 to 1.4 will follow. Let us introduce some notation. Let $M$ be a compact riemannian manifold, $d x$ the riemannian volume, $\Delta$ the Laplace-Beltrami operator, $H^{s}(M)$ the Sobolev space on $M$. Let $m \in \mathbb{R}_{+}$be a fixed given number. We define for any $s \in \mathbb{R}$ a space $E^{s}$ of functions on $\mathbb{R} \times M$ by

$$
E^{s}=\left\{u \in C^{0}\left(\mathbb{R} ; H^{s}(M)\right) ; \forall k \in \mathbb{N}, \partial_{t}^{k} u \in C^{0}\left(\mathbb{R} ; H^{s-k m}(M)\right)\right\} .
$$

For $\sigma \in \mathbb{R}$, we denote by $\mathcal{L}^{\sigma}$ a subspace of the space of operators from $C^{0}\left(\mathbb{R} ; C^{\infty}(M)\right)$ to $C^{0}\left(\mathbb{R} ; \mathcal{D}^{\prime}(M)\right)$ satisfying the following:

- Each $Q \in \mathcal{L}^{\sigma}$ is a smooth family in $t$ of bounded operators from $H^{s}(M)$ to $H^{s-\sigma}(M)$.

- If one sets $\operatorname{Ad}\left(\partial_{t}\right) Q=\left[\partial_{t}, Q\right]=\partial_{t} Q$, for each $k \in \mathbb{N}$, any $Q \in \mathcal{L}^{\sigma}, \operatorname{Ad}^{k}\left(\partial_{t}\right) Q \in \mathcal{L}^{\sigma}$ with estimates

$$
\left\|\left[\operatorname{Ad}^{k}\left(\partial_{t}\right) Q u\right](t, \cdot)\right\|_{H^{s-\sigma}} \leq C_{k, s}\|u\|_{H^{s}}
$$

uniformly for $t \in \mathbb{R}, u \in H^{s}$.

- If $Q^{*}$ denotes the adjoint of $Q \in \mathcal{L}^{\sigma}$ (at fixed time, for the usual $L^{2}$-pairing), then $Q^{*} \in \mathcal{L}^{\sigma}$.

- For any $\sigma_{1}, \sigma_{2} \in \mathbb{R}$,

$$
\mathcal{L}^{\sigma_{1}} \circ \mathcal{L}^{\sigma_{2}} \subset \mathcal{L}^{\sigma_{1}+\sigma_{2}} \text { and, when } \sigma_{1} \leq \sigma_{2}, \mathcal{L}^{\sigma_{1}} \subset \mathcal{L}^{\sigma_{2}} .
$$

We denote $\mathcal{L}^{-\infty}=\bigcap_{\sigma \in \mathbb{R}} \mathcal{L}^{\sigma}$.

Fix $\sigma_{0} \in[0, m]$ and self-adjoint operators $\Lambda \in \mathcal{L}^{m}, V \in \mathcal{L}^{\sigma_{0}}$. Consider the abstract equation

$$
\begin{aligned}
\left(i \partial_{t}+\Lambda+V\right) u & =0 \\
\left.u\right|_{t=0} & =u_{0},
\end{aligned}
$$

where $u_{0}$ is given in $H^{s}(M)$ for some $s \geq 0$. We introduce the following assumptions: For any $\sigma \in \mathbb{R}$, there are subspaces $\mathcal{L}_{\mathrm{D}}^{\sigma}, \mathcal{L}_{\mathrm{ND}}^{\sigma}$ of $\mathcal{L}^{\sigma}$, invariant under $Q \rightarrow Q^{*}$, such that

- For any $Q \in \mathcal{L}^{\sigma}$, there are $Q^{(1)} \in \mathcal{L}_{\mathrm{D}}^{\sigma}, Q^{(2)} \in \mathcal{L}_{\mathrm{ND}}^{\sigma}$ with

$$
Q=Q^{(1)}+Q^{(2)} \text {. }
$$

- There is $\rho>0$ and, for every $\sigma \in \mathbb{R}$, any $\Sigma \in \mathcal{L}_{\mathrm{ND}}^{\sigma}$, there is $Q \in \mathcal{L}_{\mathrm{ND}}^{\sigma-\rho-\sigma_{0}}$ such that

$$
[Q, \Lambda]-\Sigma \in \mathcal{L}^{-\infty} \text {. }
$$


- There is an element $\tilde{\Delta} \in \mathcal{L}^{2}$ and, for every $s \in 2 \mathbb{N}$, a constant $C>0$ such that, for any $Q \in \mathcal{L}_{\mathrm{D}}^{\sigma}$

$$
[Q, \tilde{\Delta}] \in \mathcal{L}^{-\infty},[\Lambda, \tilde{\Delta}]=0,\left[i \partial_{t}, \tilde{\Delta}\right]=0
$$

and for any $u \in E^{s}$, any $t \in \mathbb{R}$,

$$
C^{-1}\|u(t, \cdot)\|_{H^{s}}^{2} \leq\|u(t, \cdot)\|_{L^{2}}^{2}+\left\|\tilde{\Delta}^{s / 2} u(t, \cdot)\right\|_{L^{2}}^{2} \leq C\|u(t, \cdot)\|_{H^{s}}^{2} .
$$

The main result of this section is the following:

Theorem 2.1 Under assumptions (2.5) to (2.8), for any $s>0$, there is a constant $C>0$ such that, for any $u \in E^{s}$ solving equation (2.4), the bound

$$
\|u(t, \cdot)\|_{H^{s}} \leq C(1+|t|)\left\|u_{0}\right\|_{H^{s}}
$$

holds for any $t \in \mathbb{R}$.

We shall prove Theorem 2.1 rewriting equation (2.4) under an equivalent form, exploiting assumptions (2.5) and (2.6).

Proposition 2.2 Assume that (2.5) and (2.6) hold. Let $N \in \mathbb{N}^{*}$. Set $P_{0}=i \partial_{t}+\Lambda$. There are sequences $\left(Q_{j}^{\prime}\right)_{1 \leq j \leq N},\left(Q_{j}^{\prime \prime}\right)_{1 \leq j \leq N}$ satisfying

$$
\begin{gathered}
Q_{j}^{\prime} \in \mathcal{L}^{-j \rho},\left[Q_{j}^{\prime}, \Lambda\right] \in \mathcal{L}^{\sigma_{0}-(j-1) \rho},\left(Q_{j}^{\prime}\right)^{*}=-Q_{j}^{\prime} \\
Q_{j}^{\prime \prime} \in \mathcal{L}^{-(j+1) \rho},\left[Q_{j}^{\prime \prime}, \Lambda\right] \in \mathcal{L}^{\sigma_{0}-j \rho},\left(Q_{j}^{\prime \prime}\right)^{*}=Q_{j}^{\prime \prime},
\end{gathered}
$$

there are elements $V^{N} \in \mathcal{L}_{\mathrm{D}}^{\sigma_{0}}$ with $\left(V^{N}\right)^{*}=V^{N}$, there are elements

$$
\begin{aligned}
& R_{1}^{N} \in \mathcal{L}^{\sigma_{0}-N \rho},\left(R_{1}^{N}\right)^{*}=R_{1}^{N} \\
& R_{2}^{N} \in \mathcal{L}^{-(N+2) \rho} \text { with }\left[R_{2}^{N}, \Lambda\right] \in \mathcal{L}^{\sigma_{0}-(N+1) \rho},\left(R_{2}^{N}\right)^{*}=R_{2}^{N},
\end{aligned}
$$

such that, if we set $Q_{j}=Q_{j}^{\prime}+Q_{j}^{\prime \prime}, Q^{N}=\sum_{j=1}^{N} Q_{j}$

$$
\left(I+Q^{N}\right)^{*}\left(i \partial_{t}+\Lambda+V\right)\left(I+Q^{N}\right)=i \partial_{t}+\Lambda+V^{N}+R_{1}^{N}+\frac{1}{2}\left[R_{2}^{N} P_{0}+P_{0} R_{2}^{N}\right] .
$$

Before proving the proposition, let us first compute the left hand side of (2.11).

Lemma 2.3 Let $Q_{j}^{\prime}, Q_{j}^{\prime \prime}$ be given operators satisfying (2.10) for $1 \leq j \leq N$. Denote $Q^{\prime N}=$ $\sum_{j=1}^{N} Q_{j}^{\prime}, Q^{\prime \prime N}=\sum_{j=1}^{N} Q_{j}^{\prime \prime}$. One may find

- Elements $S_{j} \in \mathcal{L}^{-(j+1) \rho}, 1 \leq j \leq N+1$, such that $\left[\Lambda, S_{j}\right] \in \mathcal{L}^{\sigma_{0}-j \rho}, S_{j}^{*}=S_{j}$, depending only on $Q_{\ell}^{\prime}, 1 \leq \ell \leq j, Q_{\ell}^{\prime \prime}, 1 \leq \ell<j$,

- Elements $\tilde{S}_{j} \in \mathcal{L}^{\sigma_{0}-(j-1) \rho}, 2 \leq j \leq N+1$, depending only on $Q_{\ell}^{\prime}, Q_{\ell}^{\prime \prime}, 1 \leq \ell<j$, such that $\tilde{S}_{j}^{*}=\tilde{S}_{j}$,

so that, denoting $S^{N}=\sum_{j=1}^{N+1} S_{j}, \tilde{S}^{N}=\sum_{j=2}^{N+1} \tilde{S}_{j}$,

$$
\left(I+Q^{N}\right)^{*}\left(P_{0}+V\right)\left(I+Q^{N}\right)=P_{0}+V-\left[Q^{\prime N}, \Lambda\right]+Q^{\prime \prime N} P_{0}+P_{0} Q^{\prime \prime N}+\frac{1}{2}\left[S^{N} P_{0}+P_{0} S^{N}\right]+\tilde{S}^{N} .
$$


Proof: Using that $\left(Q^{\prime N}\right)^{*}=-Q^{\prime N},\left(Q^{\prime \prime N}\right)^{*}=Q^{\prime \prime N}$, we write

$$
\begin{aligned}
\left(I+Q^{N}\right)^{*}\left(P_{0}+V\right)\left(I+Q^{N}\right)=P_{0}+ & V-\left[Q^{\prime N}, P_{0}\right]+Q^{\prime \prime N} P_{0}+P_{0} Q^{\prime \prime N} \\
& +\frac{1}{2}\left[\left(Q^{N}\right)^{*} Q^{N} P_{0}+P_{0}\left(Q^{N}\right)^{*} Q^{N}\right] \\
+ & \frac{1}{2}\left[\left(Q^{N}\right)^{*}\left[P_{0}, Q^{N}\right]+\left[\left(Q^{N}\right)^{*}, P_{0}\right] Q^{N}\right] \\
+ & \left(Q^{N}\right)^{*} V+V Q^{N}+\left(Q^{N}\right)^{*} V Q^{N} .
\end{aligned}
$$

Let us check that the terms in the right hand side may be written as contributions to the right hand side of (2.12). We write $\left[Q^{\prime N}, P_{0}\right]$ as the sum of $\left[Q^{\prime N}, \Lambda\right]$, which gives the third term in the right hand side of $(2.12)$, and of $\left[Q^{\prime N}, i \partial_{t}\right]$ that may be written

$$
\sum_{j=2}^{N+1}\left[Q_{j-1}^{\prime}, i \partial_{t}\right]
$$

By (2.10) and the definition of $\mathcal{L}^{\sigma}$ classes, $\left[Q_{j-1}^{\prime}, i \partial_{t}\right] \in \mathcal{L}^{-(j-1) \rho} \subset \mathcal{L}^{\sigma_{0}-(j-1) \rho}$. Since this bracket is self-adjoint, it contributes to $\tilde{S}_{j}$. To study the $\left(Q^{N}\right)^{*} Q^{N} P_{0}+P_{0}\left(Q^{N}\right)^{*} Q^{N}$ contribution in (2.13), decompose

$$
\begin{aligned}
\left(Q^{N}\right)^{*} Q^{N} & =\left(Q^{\prime N}\right)^{*} Q^{\prime N}+B \\
B & =\left(Q^{\prime N}\right)^{*} Q^{\prime \prime N}+\left(Q^{\prime \prime N}\right)^{*} Q^{\prime N}+\left(Q^{\prime \prime N}\right)^{*} Q^{\prime \prime N} .
\end{aligned}
$$

Term $B$ is the sum in $j \geq 2$ of quantities

$$
\sum_{\substack{j_{1}+j_{2}=j \\ 1 \leq j_{1}, j_{2} \leq N}}\left(Q_{j_{1}}^{\prime}{ }^{*} Q_{j_{2}}^{\prime \prime}+Q_{j_{2}}^{\prime \prime}{ }^{*} Q_{j_{1}}^{\prime}\right)+\sum_{\substack{j_{1}+j_{2}=j-1 \\ 1 \leq j_{1}, j_{2} \leq N}} Q_{j_{1}}^{\prime \prime}{ }^{*} Q_{j_{2}}^{\prime \prime} .
$$

By (2.10), (2.3), this expression belongs to $\mathcal{L}^{-(j+1) \rho}$, its bracket with $\Lambda$ is in $\mathcal{L}^{\sigma_{0}-j \rho}$, and it is self-adjoint. Moreover, it depends only on $Q_{\ell}^{\prime}, Q_{\ell}^{\prime \prime}$ for $\ell<j$ and $\ell \leq N$. Consequently, it gives in (2.12) a contribution to $S_{j}$ when $2 \leq j \leq N$, and to $S_{N+1}$ when $j \geq N+1$.

Consider now the contribution of

$$
\left(Q^{\prime N}\right)^{*} Q^{\prime N} P_{0}+P_{0}\left(Q^{\prime N}\right)^{*} Q^{\prime N}
$$

to the right hand side of $(2.13)$. We write $\left(Q^{\prime N}\right)^{*} Q^{\prime N}$ as the sum for $j \geq 1$ of

$$
\sum_{\substack{j_{1}+j_{2}=j+1 \\ 1 \leq j_{1}, j_{2} \leq N}}\left(Q_{j_{1}}^{\prime}\right)^{*} Q_{j_{2}}^{\prime}
$$

This is a self-adjoint quantity, belonging to $\mathcal{L}^{-(j+1) \rho}$, whose bracket with $\Lambda$ is in $\mathcal{L}^{\sigma_{0}-j \rho}$. Moreover, it depends only on $Q_{\ell}^{\prime}, \ell \leq \min (j, N)$, so it contributes to $S_{j}$ for $j \leq N-1$, and to $S_{N}$ for $j \geq N$, in the last but one term in (2.12).

Let us study next the

$$
\left(Q^{N}\right)^{*}\left[P_{0}, Q^{N}\right]+\left[\left(Q^{N}\right)^{*}, P_{0}\right] Q^{N}
$$


term in the right hand side of (2.13). We may write (2.16) as a sum in $j \geq 2$ of

$$
\sum_{\substack{j_{1}+j_{2}=j \\ 1 \leq j_{1}, j_{2} \leq N}}\left(Q_{j_{1}}^{*}\left[P_{0}, Q_{j_{2}}\right]+\left[Q_{j_{2}}^{*}, P_{0}\right] Q_{j_{1}}\right) .
$$

Since $\left[P_{0}, Q_{j}\right]=\left[i \partial_{t}, Q_{j}\right]+\left[\Lambda, Q_{j}\right]$ belongs to $\mathcal{L}^{\sigma_{0}-(j-1) \rho}$ by $(2.10)$, and since $(2.17)$ is self-adjoint and depends only on $Q_{\ell}^{\prime}, Q_{\ell}^{\prime \prime}$ for $\ell<\inf (j, N+1)$, we get a contribution to $\tilde{S}_{j}$ when $j \leq N$, and to $\widetilde{S}_{N+1}$ when $j>N$, in the right hand side of (2.12).

We are left with examining the $V$-contributions in the right hand side of (2.13). Since $\left(Q^{N}\right)^{*} V+$ $V Q^{N}$ is the sum for $2 \leq j \leq N+1$ of $Q_{j-1}^{*} V+V Q_{j-1} \in \mathcal{L}^{\sigma_{0}-(j-1) \rho}$, and since $V=V^{*}$, we get again a contribution to $\tilde{S}_{j}$ in (2.12). The term $\left(Q^{N}\right)^{*} V Q^{N}$ is treated in a similar way. This concludes the proof of the lemma.

Proof of Proposition 2.2: Let us show that we may construct recursively a sequence of operators $Q_{1}^{\prime}, Q_{1}^{\prime \prime}, \ldots, Q_{N}^{\prime}, Q_{N}^{\prime \prime}$ so that the right hand side of $(2.11)$ may be written for $r=1, \ldots, N+1$,

$$
\begin{array}{r}
i \partial_{t}+\Lambda+V^{r-1}-\sum_{j=r}^{N}\left[Q_{j}^{\prime}, \Lambda\right]+\sum_{j=r}^{N}\left[Q_{j}^{\prime \prime} P_{0}+P_{0} Q_{j}^{\prime \prime}\right]+\frac{1}{2} \sum_{j=r}^{N+1}\left[S_{j} P_{0}+P_{0} S_{j}\right] \\
+\sum_{j=r}^{N+1} \tilde{S}_{j}+\tilde{R}_{N+1}^{r}
\end{array}
$$

where $V^{r-1} \in \mathcal{L}_{\mathrm{D}}^{\sigma_{0}},\left(V^{r-1}\right)^{*}=V^{r-1}$ for $r \geq 2, V^{0}=0, \tilde{S}_{1}=V, \tilde{R}_{N+1}^{r} \in \mathcal{L}^{\sigma_{0}-N \rho},\left(\tilde{R}_{N+1}^{r}\right)^{*}=$ $\tilde{R}_{N+1}^{r}$. Note that (2.18) with $r=1$ is the conclusion of Lemma 2.3 by our definition of $V^{0}, \tilde{S}_{1}$ if we set $\tilde{R}_{N+1}^{1}=\tilde{S}_{N+1}$. Moreover, (2.18) with $r=N+1$ is exactly the conclusion (2.11) we want to reach, setting $R_{1}^{N}=\tilde{R}_{N+1}^{N+1}, R_{2}^{N}=S_{N+1}$. Assume (2.18) has been obtained at rank $r$. Decompose according to (2.5) $\tilde{S}_{r}=V^{r,(1)}+V^{r,(2)}$ with $V^{r,(1)} \in \mathcal{L}_{\mathrm{D}}^{\sigma_{0}-(r-1) \rho}$ and $V^{r,(2)} \in \mathcal{L}_{\mathrm{ND}}^{\sigma_{0}-(r-1) \rho}$. Since $\tilde{S}_{r}^{*}=\tilde{S}_{r}$, we may assume $\left(V^{r,(1)}\right)^{*}=V^{r,(1)},\left(V^{r,(2)}\right)^{*}=V^{r,(2)}$. Moreover, these operators depend only on $Q_{\ell}^{\prime}, Q_{\ell}^{\prime \prime}, \ell<r$. We set $V^{r}=V^{r-1}+V^{r,(1)}$ and we use property $(2.6)$ to find $Q_{r}^{\prime} \in \mathcal{L}_{\mathrm{ND}}^{-r \rho}$ such that $\left[Q_{r}^{\prime}, \Lambda\right]-V^{r,(2)}$ is an element of $\mathcal{L}^{-\infty}$ that contributes to $\tilde{R}_{N+1}^{r+1}$ in (2.18) at rank $r+1$. Moreover, we may assume $\left(Q_{r}^{\prime}\right)^{*}=-Q_{r}^{\prime}$. This eliminates the $\tilde{S}_{r}$ contribution in (2.18).

We are left with finding $Q_{r}^{\prime \prime}$ satisfying (2.10), such that

$$
Q_{r}^{\prime \prime} P_{0}+P_{0} Q_{r}^{\prime \prime}=-\frac{1}{2}\left[S_{r} P_{0}+P_{0} S_{r}\right] .
$$

Since by Lemma $2.3, S_{r}$ depends only on $Q_{1}^{\prime}, \ldots, Q_{r}^{\prime}, Q_{1}^{\prime \prime}, \ldots, Q_{r-1}^{\prime \prime}$, which have been already determined, we may define $Q_{r}^{\prime \prime}=-\frac{1}{2} S_{r}$. The conditions satisfied by $S_{r}$ according to Lemma 2.3 show that $Q_{r}^{\prime \prime}$ obeys (2.10). This concludes the proof of Proposition 2.2.

Proof of Theorem 2.1: By interpolation, it is enough to prove (2.9) when $s \in 2 \mathbb{N}$. Apply Proposition 2.2 with $\rho N \geq s+m \geq s+\sigma_{0}$. We may find $P^{N}=\sum_{j=1}^{N} P_{j}$, with $P_{j} \in \mathcal{L}^{-j \rho}$, such that

$$
\left(I+Q^{N}\right)\left(I+P^{N}\right)-I=R^{N}
$$


belongs to $\mathcal{L}^{-\rho(N+1)}$. If $u$ solves $(2.4)$, we set

$$
v=\left(I+P^{N}\right) u
$$

so that, for a constant $C$ depending only on $N$ and on the index of regularity,

$$
\begin{aligned}
\|v(t, \cdot)\|_{H^{s}} & \leq C\|u(t, \cdot)\|_{H^{s}} \\
\|u(t, \cdot)\|_{H^{s}} & \leq C\left[\|v(t, \cdot)\|_{H^{s}}+\|u(t, \cdot)\|_{L^{2}}\right] \\
\left\|\partial_{t} v(t, \cdot)\right\|_{H^{\sigma}} & \leq C\left[\left\|\partial_{t} u(t, \cdot)\right\|_{H^{\sigma}}+\|u(t, \cdot)\|_{H^{\sigma}}\right](\sigma \in \mathbb{R}) .
\end{aligned}
$$

By (2.11) and (2.19) and the fact that $u$ solves (2.4),

$$
\begin{aligned}
\left(i \partial_{t}+\right. & \left.\Lambda+V^{N}+R_{1}^{N}+\frac{1}{2}\left[R_{2}^{N} P_{0}+P_{0} R_{2}^{N}\right]\right) v \\
= & \left(I+Q^{N}\right)^{*}\left[i \partial_{t}+\Lambda+V, R^{N}\right] u \stackrel{\text { def }}{=} f .
\end{aligned}
$$

By (2.2), (2.3) and the choice of $N$

$$
\|f(t, \cdot)\|_{H^{s}} \leq C\|u(t, \cdot)\|_{L^{2}}
$$

for some uniform constant $C>0$. Consequently

$$
\left(i \partial_{t}+\Lambda+V^{N}\right) v=g
$$

where by (2.2), (2.3) and the last inequality (2.20)

$$
\|g(t, \cdot)\|_{H^{s}} \leq C\left[\|u(t, \cdot)\|_{L^{2}}+\|v(t, \cdot)\|_{L^{2}}\right] .
$$

By commutation condition (2.7), and the fact that $V^{N} \in \mathcal{L}_{\mathrm{D}}^{\sigma_{0}}$, we may write

$$
\left(i \partial_{t}+\Lambda+V^{N}\right)\left(\tilde{\Delta}^{s / 2} v\right)=w
$$

with $\|w(t, \cdot)\|_{L^{2}} \leq C\left[\|u(t, \cdot)\|_{L^{2}}+\|v(t, \cdot)\|_{L^{2}}\right]$. The $L^{2}$-energy inequality - which holds since at fixed $t, \Lambda, V^{N}$ are self-adjoint - together with (2.8) implies that for any $t \geq 0$

$$
\|v(t, \cdot)\|_{H^{s}} \leq C\left[\|v(0, \cdot)\|_{H^{s}}+\int_{0}^{t}\left[\|u(\tau, \cdot)\|_{L^{2}}+\|v(\tau, \cdot)\|_{L^{2}}\right] d \tau\right] .
$$

Since by $(2.20),\|v(\tau, \cdot)\|_{L^{2}} \leq C\|u(\tau, \cdot)\|_{L^{2}}$ and using the conservation of the $L^{2}$-norm for $(2.4)$ $\|u(\tau, \cdot)\|_{L^{2}}=\|u(0, \cdot)\|_{L^{2}}$, we obtain

$$
\|v(t, \cdot)\|_{H^{s}} \leq C\left[\|v(0, \cdot)\|_{H^{s}}+\|u(0, \cdot)\|_{L^{2}} t\right]
$$

for any $t \geq 0$. Estimate (2.9) follows from that and (2.20). 


\section{$3 \quad$ Schrödinger equation on $\mathbb{T}^{d}$}

We assume here that the manifold $M$ is the torus $\mathbb{T}^{d}=(\mathbb{R} / 2 \pi \mathbb{Z})^{d}$. Let $V: \mathbb{R} \times \mathbb{T}^{d} \rightarrow \mathbb{R}$ be a $C^{\infty}$ function of $(t, x)$, bounded as well as all its derivatives, and let $u$ be a solution of (1.1). For $n \in \mathbb{Z}^{d}$, we denote by $\Pi_{n}$ the spectral projector

$$
\Pi_{n} u=\left\langle u, \frac{e^{i n x}}{(2 \pi)^{d / 2}}\right\rangle \frac{e^{i n x}}{(2 \pi)^{d / 2}} .
$$

Definition 3.1 For $\sigma \in \mathbb{R}$, we denote by $\mathcal{L}^{\sigma}$ the space of smooth families in time of continuous operators from $C^{\infty}\left(\mathbb{T}^{d}\right)$ to $\mathcal{D}^{\prime}\left(\mathbb{T}^{d}\right)$, such that, for any $k, N \in \mathbb{N}$, there is $C>0$ with

$$
\left\|\Pi_{n} \partial_{t}^{k} Q(t, \cdot) \Pi_{n^{\prime}}\right\|_{\mathcal{L}\left(L^{2}\right)} \leq C\left(1+|n|+\left|n^{\prime}\right|\right)^{\sigma}\left\langle n-n^{\prime}\right\rangle^{-N}
$$

for any $n, n^{\prime} \in \mathbb{Z}^{d}$, any $t \in \mathbb{R}\left(\right.$ where $\left.\langle k\rangle=\left(1+|k|^{2}\right)^{1 / 2}\right)$.

The above definition implies immediately that conditions (2.2) and (2.3) are satisfied by the class $\mathcal{L}^{\sigma}$. Moreover, $\Lambda=-\Delta$ belongs to $\mathcal{L}^{2}$, and the operator of multiplication by $V$ is in $\mathcal{L}^{0}$, as follows from Fourier series decomposition of $x \rightarrow V(t, x)$ at every fixed $t$. We shall consequently apply the results of section 2 with $m=2, \sigma_{0}=0$. Let us state the following lemma due to Bourgain ([4] Lemma 8.1; see also for the proof lemma 19.10 in [6]):

Lemma 3.2 (Bourgain) For any $\delta \in] 0, \frac{1}{10}[$, there are $\rho \in] 0, \delta\left[, \gamma>0\right.$ and a partition $\left(\Omega_{\alpha}\right)_{\alpha \in A}$ of $\mathbb{Z}^{d}$ such that

$$
\begin{array}{r}
\forall \alpha \in A, \forall n \in \Omega_{\alpha}, \forall n^{\prime} \in \Omega_{\alpha},\left|n-n^{\prime}\right|+\left.|| n\right|^{2}-\left.\left|n^{\prime}\right|^{2}|<\gamma+| n\right|^{\delta}, \\
\forall \alpha, \beta \in A, \alpha \neq \beta, \forall n \in \Omega_{\alpha}, \forall n^{\prime} \in \Omega_{\beta},\left|n-n^{\prime}\right|+\left.|| n\right|^{2}-\left.\left|n^{\prime}\right|^{2}|>| n\right|^{\rho} .
\end{array}
$$

We denote for $\alpha \in A$

$$
\widetilde{\Pi}_{\alpha}=\sum_{n \in \Omega_{\alpha}} \Pi_{n}
$$

Definition 3.3 We denote by $\mathcal{L}_{\mathrm{D}}^{\sigma}$ (resp. $\mathcal{L}_{\mathrm{ND}}^{\sigma}$ ) the subspace of $\mathcal{L}^{\sigma}$ given by those operators $Q \in \mathcal{L}^{\sigma}$ such that for any $\alpha, \beta \in A$ with $\alpha \neq \beta$ (resp. any $\alpha \in A$ ) $\widetilde{\Pi}_{\alpha} Q \widetilde{\Pi}_{\beta} \equiv 0$ (resp. $\left.\widetilde{\Pi}_{\alpha} Q \widetilde{\Pi}_{\alpha} \equiv 0\right)$.

Proof of Theorem 1.1: To deduce Theorem 1.1 from Theorem 2.1, we just need to check that conditions (2.5) to (2.8) are satisfied by the class of operators we just defined. Take $Q \in \mathcal{L}^{\sigma}$ and define

$$
Q^{(1)}=\sum_{\alpha \in A} \widetilde{\Pi}_{\alpha} Q \widetilde{\Pi}_{\alpha} \in \mathcal{L}_{\mathrm{D}}^{\sigma}, Q^{(2)}=\sum_{\substack{\alpha, \beta \in A \\ \alpha \neq \beta}} \widetilde{\Pi}_{\alpha} Q \widetilde{\Pi}_{\beta} \in \mathcal{L}_{\mathrm{ND}}^{\sigma} .
$$


Then (2.5) holds true. Consider now $\Sigma \in \mathcal{L}_{\text {ND }}^{\sigma}$ and let us find $Q \in \mathcal{L}_{\text {ND }}^{\sigma-\rho}$ solving (2.6) i.e. $-[Q, \Delta]-\Sigma \in \mathcal{L}^{-\infty}$. Write $\Sigma=\Sigma_{1}+R$ with

$$
\Sigma_{1}=\sum_{n \in \mathbb{Z}^{d}} \sum_{n^{\prime} \in \mathbb{Z}^{d}} \mathbb{1}_{\left\{\|\left. n\right|^{2}-\left|n^{\prime}\right|^{2} \mid>\frac{1}{4}\left(|n|+\left|n^{\prime}\right|\right)^{\rho}\right\}} \Pi_{n} \Sigma \Pi_{n^{\prime}}
$$

where $\rho$ is given by Lemma 3.2 when some $\delta \in] 0, \frac{1}{10}[$ has been fixed. Since the equation $-[Q, \Delta]=$ $\Sigma_{1}$ may be written

$$
\left(\left|n^{\prime}\right|^{2}-|n|^{2}\right) \Pi_{n} Q \Pi_{n^{\prime}}=\Pi_{n} \Sigma_{1} \Pi_{n^{\prime}}
$$

we see that (3.5) defines an element $Q \in \mathcal{L}_{\text {ND }}^{\sigma-\rho}$. We are left with checking that $R=\Sigma-\Sigma_{1} \in \mathcal{L}^{-\infty}$. But

$$
\Pi_{n} R \Pi_{n^{\prime}}=\mathbb{1}_{\left\{\left.|| n\right|^{2}-\left|n^{\prime}\right|^{2} \mid \leq \frac{1}{4}\left(|n|+\left|n^{\prime}\right|\right)^{\rho}\right\}} \Pi_{n} \Sigma \Pi_{n^{\prime}}
$$

and since $\Sigma \in \mathcal{L}_{\mathrm{ND}}^{\sigma}$, this expression is non zero only when $n$ and $n^{\prime}$ belong to $\Omega_{\alpha}$ and $\Omega_{\beta}$ with $\alpha \neq \beta$. So the second condition in (3.2), together with the cut-off in (3.6), implies that $\left|n-n^{\prime}\right| \geq c\left(|n|+\left|n^{\prime}\right|\right)^{\rho}$ for some $c>0$. It follows from (3.1) that $R \in \mathcal{L}^{-\infty}$.

We still have to check conditions (2.7) and (2.8). For any $\alpha \in A$, we choose $n(\alpha) \in \Omega_{\alpha}$ and define

$$
\tilde{\Delta} u=\sum_{\alpha \in A}|n(\alpha)|^{2} \widetilde{\Pi}_{\alpha} u
$$

It follows from the definition of $\mathcal{L}_{\mathrm{D}}^{\sigma}$ that the first condition $(2.7)$ holds (actually $[\tilde{\Delta}, Q]=0$ for $\left.Q \in \mathcal{L}_{\mathrm{D}}^{\sigma}\right)$. The second and third conditions are trivially verified. Finally, by the first condition in (3.2), there is $C>0$ such that for any $\alpha \in A$

$$
C^{-1}\left(1+|n(\alpha)|^{2}\right)\left\|\widetilde{\Pi}_{\alpha} u\right\|_{L^{2}}^{2} \leq \sum_{n \in \Omega_{\alpha}}\left(1+|n|^{2}\right)\left\|\Pi_{n} u\right\|_{L^{2}}^{2} \leq C\left(1+|n(\alpha)|^{2}\right)\left\|\widetilde{\Pi}_{\alpha} u\right\|_{L^{2}}^{2}
$$

which shows that (2.8) holds. Consequently, we may apply Theorem 2.1, which brings Theorem 1.1.

\section{Schrödinger equation on Zoll manifolds}

We assume now that $M$ is a compact Zoll manifold. By results of Colin de Verdière [7] (see also Duistermaat-Guillemin [10], Guillemin [12] and Weinstein [15]), it is known that if $\tau$ is the minimal period of the geodesic flow, the eigenvalues of $\sqrt{-\Delta}$ on $M$ are contained in $\bigcup_{n=0}^{+\infty} I_{n}$, where for $n \geq 1$

$$
I_{n}=\left[\frac{2 \pi}{\tau} n+\alpha-\frac{C}{n_{0}+n}, \frac{2 \pi}{\tau} n+\alpha+\frac{C}{n_{0}+n}\right]
$$

for some $\alpha \in \mathbb{R}$, some $C>0$, some large $n_{0} \in \mathbb{N}$, and where $I_{0}$ is a finite set of positive numbers strictly smaller than $\frac{2 \pi}{\tau}+\alpha-\frac{C}{n_{0}+1}$. If $n_{0}$ is large enough, these intervals are disjoint. For any $n \in \mathbb{N}$, we define $\Pi_{n}$ to be the spectral projector associated to $I_{n}$. 
Let $V: \mathbb{R} \times M \rightarrow \mathbb{R}$ a $C^{\infty}$ function of $(t, x)$, bounded as well as its derivatives. We consider again equation (1.1).

Definition 4.1 For $\sigma \in \mathbb{R}$, we denote by $\mathcal{L}^{\sigma}$ the space of smooth families in time of continuous operators from $C^{\infty}(M)$ to $\mathcal{D}^{\prime}(M)$ such that for any $k, N \in \mathbb{N}$, there is $C>0$ with

$$
\left\|\Pi_{n} \partial_{t}^{k} Q(t, \cdot) \Pi_{n^{\prime}}\right\|_{\mathcal{L}\left(L^{2}\right)} \leq C\left(1+n+n^{\prime}\right)^{\sigma}\left\langle n-n^{\prime}\right\rangle^{-N}
$$

for any $n, n^{\prime} \in \mathbb{N}$, any $t \in \mathbb{R}$.

Conditions (2.2) and (2.3) are satisfied by elements of this class, as it follows from the characterization of Sobolev spaces in terms of the spectral projectors $\Pi_{n}$ that

$$
\|u\|_{H^{s}}^{2} \sim \sum_{n=0}^{+\infty}\left(1+|n|^{2}\right)^{s}\left\|\Pi_{n} u\right\|_{L^{2}}^{2} .
$$

We shall set $\Lambda=-\Delta \in \mathcal{L}^{2}$ (so $m=2$ with the notations of section 2), and the operator of multiplication by $V$ is in $\mathcal{L}^{0}$. Actually, if we set $\lambda_{n}=\frac{2 \pi}{\tau} n+\alpha, n \in \mathbb{N}^{*}, \lambda_{0}=1$, there is a constant $C_{0}>0$ and, for every $n \in \mathbb{N}$, an endomorphism $A_{n}$ of the range of $\Pi_{n}$, whose operator norm is bounded by $C_{0}$, such that

$$
-\Delta \Pi_{n}=\lambda_{n}^{2} \Pi_{n}+A_{n} \Pi_{n} .
$$

Consequently

$$
\left(\lambda_{n}^{2}-\lambda_{n^{\prime}}^{2}\right) \Pi_{n} V \Pi_{n^{\prime}}=-A_{n} \Pi_{n} V \Pi_{n^{\prime}}+\Pi_{n} V \Pi_{n^{\prime}} A_{n^{\prime}}-\Pi_{n}[\Delta, V] \Pi_{n^{\prime}},
$$

whence

$$
\left|\lambda_{n}^{2}-\lambda_{n^{\prime}}^{2}\right|\left\|\Pi_{n} V \Pi_{n^{\prime}}\right\|_{\mathcal{L}\left(L^{2}\right)} \leq C\left(1+\lambda_{n}+\lambda_{n^{\prime}}\right)
$$

since $[\Delta, V]$ is of order 1. Iterating (4.3), one obtains estimates of form (4.2) with $\sigma=0$.

We shall apply the abstract results of section 2 with $m=2, \sigma_{0}=0, \rho=1$. Let us introduce some notation. For $N_{0}$ an integer to be chosen below large enough, we set

$$
\begin{aligned}
\widetilde{\Pi}_{0} & =\sum_{n=0}^{N_{0}} \Pi_{n}, \widetilde{\Pi}_{n}=\Pi_{n} \text { if } n \geq N_{0}+1 \\
\Delta_{0} & =-\widetilde{\Pi}_{0}+\left(I-\widetilde{\Pi}_{0}\right) \Delta \\
-\tilde{\Delta} & =\widetilde{\Pi}_{0}+\sum_{n=N_{0}+1}^{+\infty}\left(\frac{2 \pi}{\tau} n+\alpha\right)^{2} \widetilde{\Pi}_{n} .
\end{aligned}
$$

Our assumptions made after (4.1) imply that $\frac{2 \pi}{\tau} n+\alpha \geq 0$ for any $n \in \mathbb{N}^{*}$. Moreover (4.1) implies that $\Delta_{0}-\tilde{\Delta}$ is bounded on $L^{2}$.

Definition 4.2 We define $\mathcal{L}_{\mathrm{D}}^{\sigma}$ (resp. $\mathcal{L}_{\mathrm{ND}}^{\sigma}$ ) as the subspace of $\mathcal{L}^{\sigma}$ given by those operators $Q \in \mathcal{L}^{\sigma}$ such that for any $n, n^{\prime}$ in $\mathbb{N}_{0}=\{0\} \cup\left\{n \in \mathbb{N} ; n>N_{0}\right\}$ with $n \neq n^{\prime}$ (resp. for any $\left.n \in \mathbb{N}_{0}\right) \widetilde{\Pi}_{n} Q \widetilde{\Pi}_{n^{\prime}}=0$ (resp. $\widetilde{\Pi}_{n} Q \widetilde{\Pi}_{n}=0$ ). 
Proof of Theorem 1.2: We have again to check conditions (2.5) to (2.8). If $Q \in \mathcal{L}^{\sigma}$, we decompose it as $Q=Q^{(1)}+Q^{(2)}$ with

$$
Q^{(1)}=\sum_{n \in \mathbb{N}_{0}} \widetilde{\Pi}_{n} Q \widetilde{\Pi}_{n}, Q^{(2)}=\sum_{\substack{n, n^{\prime} \in \mathbb{N}_{0} \\ n \neq n^{\prime}}} \widetilde{\Pi}_{n} Q \widetilde{\Pi}_{n^{\prime}}
$$

obtaining $Q^{(1)} \in \mathcal{L}_{\mathrm{D}}^{\sigma}, Q^{(2)} \in \mathcal{L}_{\mathrm{ND}}^{\sigma}$. Next we have to establish (2.6) with $\Lambda=-\Delta$. Since $\Delta-\Delta_{0} \in \mathcal{L}^{-\infty}$, we just need to solve for $\Sigma \in \mathcal{L}_{\mathrm{ND}}^{\sigma},\left[\Delta_{0}, Q\right]=\Sigma$. Write this equation

$$
[\tilde{\Delta}, Q]+\left[\left(\Delta_{0}-\tilde{\Delta}\right), Q\right]=\Sigma .
$$

Setting as above $\lambda_{n}=\frac{2 \pi}{\tau} n+\alpha$ for $n \geq N_{0}+1, \lambda_{0}=1$, define for $Q \in \mathcal{L}_{\mathrm{ND}}^{\sigma-1}, \Sigma \in \mathcal{L}_{\mathrm{ND}}^{\sigma}$

$$
\begin{array}{r}
L_{0}(Q)=[\tilde{\Delta}, Q]=\sum_{\substack{n, n^{\prime} \in \mathbb{N}_{0} \\
n \neq n^{\prime}}}\left(\lambda_{n^{\prime}}^{2}-\lambda_{n}^{2}\right) \widetilde{\Pi}_{n} Q \widetilde{\Pi}_{n^{\prime}} \\
L_{0}^{-1}(\Sigma)=\sum_{\substack{n, n^{\prime} \in \mathbb{N}_{0} \\
n \neq n^{\prime}}}\left(\lambda_{n^{\prime}}^{2}-\lambda_{n}^{2}\right)^{-1} \widetilde{\Pi}_{n} \Sigma \widetilde{\Pi}_{n^{\prime}}
\end{array}
$$

so that $L_{0}^{-1}(\Sigma) \in \mathcal{L}_{\mathrm{ND}}^{\sigma-1}$ and $L_{0} \circ L_{0}^{-1}(\Sigma)=\Sigma$. We define

$$
A(Q)=-\left[\Delta_{0}-\tilde{\Delta}, Q\right] .
$$

By construction

$$
\widetilde{\Pi}_{n} A(Q) \widetilde{\Pi}_{n^{\prime}}=A\left(\widetilde{\Pi}_{n} Q \widetilde{\Pi}_{n^{\prime}}\right)
$$

vanishes if $n=n^{\prime}=0$. Moreover, since for $n \in \mathbb{N}_{0}\left\|\left(\Delta_{0}-\tilde{\Delta}\right) \widetilde{\Pi}_{n}\right\|_{\mathcal{L}\left(L^{2}\right)} \leq C$, we get

$$
\left\|\widetilde{\Pi}_{n} A(Q) \widetilde{\Pi}_{n^{\prime}}\right\|_{\mathcal{L}\left(L^{2}\right)} \leq C\left\|\widetilde{\Pi}_{n} Q \widetilde{\Pi}_{n^{\prime}}\right\|_{\mathcal{L}\left(L^{2}\right)}
$$

for some uniform constant $C>0$. By (4.6), we get for any $n, n^{\prime} \in \mathbb{N}_{0}, n \neq n^{\prime}$

$$
\left\|\widetilde{\Pi}_{n} L_{0}^{-1} \circ A(Q) \widetilde{\Pi}_{n^{\prime}}\right\|_{\mathcal{L}\left(L^{2}\right)} \leq \frac{C}{n+n^{\prime}+1}\left\|\widetilde{\Pi}_{n} Q \widetilde{\Pi}_{n^{\prime}}\right\|_{\mathcal{L}\left(L^{2}\right)},
$$

and since we have seen that by (4.7) the left hand side vanishes when $n=n^{\prime}=0$, we may assume $n+n^{\prime} \geq N_{0}$. If this integer is large enough, we get

$$
\left\|\widetilde{\Pi}_{n} L_{0}^{-1} \circ A(Q) \widetilde{\Pi}_{n^{\prime}}\right\|_{\mathcal{L}\left(L^{2}\right)} \leq \frac{1}{2}\left\|\widetilde{\Pi}_{n} Q \widetilde{\Pi}_{n^{\prime}}\right\|_{\mathcal{L}\left(L^{2}\right)} .
$$

Define for $N \geq 2, \mathcal{L}_{\mathrm{ND}}^{\sigma}(N)$ to be the space of those $Q$ satisfying estimate (4.2) with the exponent $N$, and for $k \leq N$. This is a Banach space for the norm $\|Q\|_{\mathcal{L}_{\mathrm{ND}}^{\sigma}(N)}$ given by the best constant $C$ in the right hand side of (4.2). Estimate (4.9) shows that $L_{0}^{-1} \circ A$ is a bounded operator from $\mathcal{L}_{\mathrm{ND}}^{\sigma-1}(N)$ to itself, with operator norm smaller than $\frac{1}{2}$. Consequently, the series $\sum_{k=0}^{+\infty}\left(L_{0}^{-1} \circ A\right)^{k}$ converges in $\mathcal{L}_{\mathrm{ND}}^{\sigma-1}(N)$ for any $N \geq 2$. Write equation (4.5) as

$$
L_{0}(Q)-A(Q)=\Sigma
$$


and invert it by

$$
Q=\left(I d-L_{0}^{-1} \circ A\right)^{-1} L_{0}^{-1} \Sigma .
$$

By (4.6) $L_{0}^{-1} \Sigma \in \mathcal{L}_{\mathrm{ND}}^{\sigma-1}$, and by the above remarks, the series

$$
\sum_{k=0}^{+\infty}\left(L_{0}^{-1} \circ A\right)^{k} L_{0}^{-1} \Sigma
$$

defines an operator in $\mathcal{L}_{\mathrm{ND}}^{\sigma-1}$. This establishes property (2.6). Finally, if $Q \in \mathcal{L}_{\mathrm{D}}^{\sigma},[Q, \tilde{\Delta}]=$ 0 because of the definition of $\tilde{\Delta}$. Moreover, the last two properties in (2.7) hold trivially. Inequalities (2.8) follow from the spectral characterization of Sobolev spaces. We may therefore apply Theorem 2.1 in our framework, and get Theorem 1.2 as a consequence.

\section{Schrödinger equation on surfaces of revolution}

We assume in this section that $M=\mathbb{S}^{2}$ with a metric $g$ satisfying the assumptions of Theorem 1.3. Let us recall results of [9], mainly Theorem 6.1 of that paper. Under the preceding assumptions, there are two first order commuting self-adjoint pseudo-differential operators $Q_{1}, Q_{2}$ satisfying the following:

- The joint spectrum of $\left(Q_{1}, Q_{2}\right)$ is

$$
\Lambda_{0}=\left\{n=\left(n_{1}, n_{2}\right) \in\left(\mathbb{Z}+\frac{1}{2}\right) \times \mathbb{Z} ;\left|n_{2}\right| \leq n_{1}-\frac{1}{2}\right\} .
$$

In particular, there is an Hilbert basis $\left(\varphi_{n}\right)_{n \in \Lambda_{0}}$ of $L^{2}(M)$ made of joint eigenfunctions of $\left(Q_{1}, Q_{2}\right)$ i.e.

$$
Q_{1} \varphi_{n}=n_{1} \varphi_{n}, Q_{2} \varphi_{n}=n_{2} \varphi_{n}
$$

- There is a classical symbol of order $2, F$, defined on $\mathbb{R}^{2}$, with an asymptotic expansion in homogeneous components $F=F_{2}+F_{0}+F_{-1}+\cdots$ where $F_{2}$ is elliptic (on a conic neighborhood of $\left.\Lambda_{0}\right)$, such that $-\Delta=F\left(Q_{1}, Q_{2}\right)$. In particular

$$
-\Delta \varphi_{n}=F(n) \varphi_{n}, n \in \Lambda_{0} .
$$

For $n \in \Lambda_{0}, u \in \mathcal{D}^{\prime}(M)$, we set $\Pi_{n} u=\left\langle u, \varphi_{n}\right\rangle \varphi_{n}$.

Definition 5.1 For $\sigma \in \mathbb{R}$, we denote by $\mathcal{L}^{\sigma}$ the space of smooth families in time of continuous operators from $C^{\infty}(M)$ to $\mathcal{D}^{\prime}(M)$ such that, for any $k, N \in \mathbb{N}$, there is $C>0$ with

$$
\left\|\Pi_{n} \partial_{t}^{k} Q(t, \cdot) \Pi_{n^{\prime}}\right\|_{\mathcal{L}\left(L^{2}\right)} \leq C\left(1+|n|+\left|n^{\prime}\right|\right)^{\sigma}\left\langle n-n^{\prime}\right\rangle^{-N}
$$

for any $n, n^{\prime} \in \Lambda_{0}$. 
It follows from (5.4) and the fact that $\|u\|_{H^{s}}^{2} \sim \sum_{n \in \Lambda_{0}}\langle n\rangle^{2 s}\left\|\Pi_{n} u\right\|_{L^{2}}^{2}$ that (2.2) and (2.3) hold. Moreover $-\Delta \in \mathcal{L}^{2}$. If $(t, x) \rightarrow V(t, x)$ is a smooth function on $\mathbb{R} \times M$, with all its $(t, x)$ derivatives bounded, we see by iteration of the equality following from (5.2)

$$
\left(n_{j}-n_{j}^{\prime}\right) \Pi_{n} V \Pi_{n^{\prime}}=\Pi_{n}\left[Q_{j}, V\right] \Pi_{n^{\prime}}, j=1,2,
$$

that the operator of multiplication by $V$ is in $\mathcal{L}^{0}$. We shall apply the results of section 2 to $\Lambda=-\Delta, m=2, \sigma_{0}=0$. Let us check (2.5) to (2.8). We need to define decomposition (2.5). Let us introduce some notation. For $\left.\rho \in] 0, \frac{1}{2}\right]$, define for $\ell \in \mathbb{Z}^{2}-\{0\}$

$$
\Gamma_{\ell}=\left\{n \in \Lambda_{0} ;\left|F^{\prime}(n) \cdot \ell\right|<\rho|\ell||n|^{\rho}\right\} .
$$

We shall use the following properties of the above sets:

Lemma 5.2 (i) There is $K>0$ such that for any $\left.\rho \in] 0, \frac{1}{2}\right]$, for any $\ell \in \mathbb{Z}^{2}-\{0\}$, any $n \in \Gamma_{\ell}$ with $|n| \geq K$,

$$
\left|n \cdot \frac{\ell^{\perp}}{|\ell|}\right| \geq \frac{1}{K}|n|
$$

if $\ell^{\perp}=\left(-\ell_{2}, \ell_{1}\right)$ when $\ell=\left(\ell_{1}, \ell_{2}\right)$.

(ii) There is $K>0$ and for any $\left.\rho \in] 0, \frac{1}{2}\right]$, for any $\ell, \ell^{\prime} \in \mathbb{Z}^{2}-\{0\}$ which are not collinear

$$
\Gamma_{\ell} \cap \Gamma_{\ell^{\prime}} \subset\left\{n \in \Lambda_{0} ;|n| \leq K\left(|\ell|^{2}+\left|\ell^{\prime}\right|^{2}\right)^{\frac{1}{1-\rho}}\right\} .
$$

Proof: (i) For $|n| \geq 1$, we write $F(n)=F_{2}(n)+\tilde{F}(n)$ where $\tilde{F}$ is a symbol of order zero and $F_{2}$ is homogeneous of degree two and elliptic. For $0<|\lambda|<\frac{1}{2}$, compute

$$
F_{2}((1+\lambda) n)=(1+\lambda)^{2} F_{2}(n)=F_{2}(n)+F_{2}^{\prime}(n) \cdot(\lambda n)+O\left(|\lambda n|^{2}\right) .
$$

Then

$$
F_{2}^{\prime}(n) \cdot n=(2+\lambda) F_{2}(n)+O\left(|\lambda||n|^{2}\right),
$$

whence taking $\lambda$ small enough, $\left|F_{2}^{\prime}(n) \cdot n\right| \geq c|n|^{2}$, which implies for $|n| \geq K$ large enough

$$
\left|F^{\prime}(n) \cdot n\right| \geq c|n|^{2}
$$

for some $c>0$. If $n \in \Gamma_{\ell}$, decompose

$$
F^{\prime}(n)=A_{\ell}(n) \frac{\ell}{|\ell|}+B_{\ell}(n) \frac{\ell^{\perp}}{|\ell|}
$$

with $A_{\ell}(n)=F^{\prime}(n) \cdot \frac{\ell}{|\ell|}, B_{\ell}(n)=F^{\prime}(n) \cdot \frac{\ell^{\perp}}{|\ell|}$. If we use (5.7), estimate $\left|A_{\ell}(n)\right|$ from the definition of $\Gamma_{\ell}$, and $\left|B_{\ell}(n)\right|$ by $\left|F^{\prime}(n)\right| \leq C|n|$, we get

$$
c|n|^{2} \leq \rho|n|^{1+\rho}+C|n|\left|\frac{\ell^{\perp}}{|\ell|} \cdot n\right|
$$


for $|n| \geq K$. Enlarging $K$ if necessary, we get (5.6).

(ii) Let $n \in \Gamma_{\ell} \cap \Gamma_{\ell^{\prime}}$ and assume $|n|>K\left(|\ell|^{2}+\left|\ell^{\prime}\right|^{2}\right)^{\frac{1}{1-\rho}}$ for some $K>0$. If $K$ is large enough, (5.7) implies that $\left|F^{\prime}(n)\right| \geq c|n|$ and we may define $e=\frac{F^{\prime}(n)}{\left|F^{\prime}(n)\right|}$. Decompose $\ell=(\ell \cdot e) e+\left(\ell \cdot e^{\perp}\right) e^{\perp}$, so that

$$
\operatorname{det}\left(\ell, \ell^{\prime}\right)=(\ell \cdot e) \operatorname{det}\left(e, \ell^{\prime}\right)+\operatorname{det}(\ell, e)\left(\ell^{\prime} \cdot e\right)
$$

By definition of $\Gamma_{\ell}, \Gamma_{\ell^{\prime}}, e$,

$$
|\ell \cdot e|<\frac{\rho}{c}|\ell||n|^{\rho-1},\left|\ell^{\prime} \cdot e\right|<\frac{\rho}{c}\left|\ell^{\prime}\right||n|^{\rho-1} .
$$

Since $\operatorname{det}\left(\ell, \ell^{\prime}\right) \in \mathbb{Z}^{*}$, we deduce from (5.8)

$$
1 \leq \frac{2 \rho}{c}|\ell|\left|\ell^{\prime}\right||n|^{\rho-1}
$$

which contradicts the assumption on $n$ if $K>\left(\frac{\rho}{c}\right)^{\frac{1}{1-\rho}}$. This concludes the proof of the lemma.

Let us define the subspaces $\mathcal{L}_{\mathrm{D}}^{\sigma}$ and $\mathcal{L}_{\mathrm{ND}}^{\sigma}$. Denote by $\mathcal{P}$ a subset of $\mathbb{Z}^{2}-\{0\}$ such that for any $\ell \in \mathbb{Z}^{2}-\{0\}$, there is a unique $\ell_{0} \in \mathcal{P}$ and a unique $d \in \mathbb{Z}^{*}$ with $\ell=d \ell_{0}$, and such that if $\ell \in \mathcal{P}$, $\Gamma_{\ell} \neq \emptyset$. Fix a number $\left.\left.\rho \in\right] 0, \frac{1}{2}\right]$.

Definition 5.3 Let $A>0$ be a large constant to be chosen, $\sigma \in \mathbb{R}$.

(i) We denote by $\mathcal{L}_{\mathrm{ND}}^{\sigma}$ the subspace of those $Q \in \mathcal{L}^{\sigma}$ such that

$$
\left|n-n^{\prime}\right|<\frac{1}{A}|n|^{\rho / 2} \text { and }\left|F(n)-F\left(n^{\prime}\right)\right|>\frac{1}{A}|n|^{\rho}
$$

if $\Pi_{n} Q \Pi_{n^{\prime}} \neq 0$.

(ii) We denote by $\mathcal{L}_{\mathrm{D}}^{\sigma}$ the subspace of those $Q \in \mathcal{L}^{\sigma}$ that may be written

$$
Q=Q_{0}+\sum_{\ell \in \mathcal{P}} Q_{\ell}+R
$$

where $R \in \mathcal{L}^{-\infty}, Q_{0}$ satisfies $\Pi_{n} Q_{0} \Pi_{n^{\prime}}=0$ if $n, n^{\prime} \in \Lambda_{0}, n \neq n^{\prime}$, and for $\ell \in \mathcal{P}, \Pi_{n} Q_{\ell} \Pi_{n^{\prime}}$ is non zero only when

$$
n \in \Gamma_{\ell}, n^{\prime} \in \Gamma_{\ell},|\ell| \leq\left|n-n^{\prime}\right|<\frac{1}{A}|n|^{\rho} \text { and } n-n^{\prime} \text { is collinear to } \ell .
$$

Let us check that condition (2.5) is satisfied:

Lemma 5.4 Assume that $A$ in (5.9) is large enough relatively to $\frac{1}{\rho}$. For any $Q \in \mathcal{L}^{\sigma}$ there are $Q^{(1)} \in \mathcal{L}_{\mathrm{D}}^{\sigma}, Q^{(2)} \in \mathcal{L}_{\mathrm{ND}}^{\sigma}$ such that $Q=Q^{(1)}+Q^{(2)}$. 
Proof: Let us define

$$
Q^{(2)}=\sum_{n \in \Lambda_{0}} \sum_{n^{\prime} \in \Lambda_{0}} \mathbb{1}_{\left\{\left|n-n^{\prime}\right|<\frac{1}{A}|n|^{\rho / 2},\left|F(n)-F\left(n^{\prime}\right)\right|>\frac{1}{A}|n|^{\rho}\right\}} \Pi_{n} Q \Pi_{n^{\prime}} .
$$

Then $Q^{(2)} \in \mathcal{L}_{\mathrm{ND}}^{\sigma}$. Set

$$
R=\sum_{n \in \Lambda_{0}} \sum_{n^{\prime} \in \Lambda_{0}} \mathbb{1}_{\left\{\left|n-n^{\prime}\right| \geq \frac{1}{A}|n|^{\rho / 2}\right\}} \Pi_{n} Q \Pi_{n^{\prime}}
$$

so that $R \in \mathcal{L}^{-\infty} \subset \mathcal{L}_{\mathrm{D}}^{\sigma}$. Consequently, we may write

$$
Q-Q^{(2)}-R=\sum_{n \in \Lambda_{0}} \sum_{n^{\prime} \in \Lambda_{0}} \mathbb{1}_{\Gamma}\left(n, n^{\prime}\right) \Pi_{n} Q \Pi_{n^{\prime}}
$$

where

$$
\Gamma=\left\{\left(n, n^{\prime}\right) \in \mathbb{Z}^{2} \times \mathbb{Z}^{2} ;\left|n-n^{\prime}\right|<\frac{1}{A}|n|^{\rho / 2},\left|F(n)-F\left(n^{\prime}\right)\right| \leq \frac{1}{A}|n|^{\rho}\right\} .
$$

In other words,

$$
Q-Q^{(2)}-R=\sum_{\ell \in \mathcal{P}} \sum_{d \in \mathbb{Z}^{*}} \sum_{n \in \Lambda_{0}} \mathbb{1}_{\Gamma}(n, n+d \ell) \mathbb{1}_{\Lambda_{0}}(n+d \ell) \Pi_{n} Q \Pi_{n+d \ell}+\sum_{n \in \Lambda_{0}} \Pi_{n} Q \Pi_{n}
$$

and we define

$$
\begin{aligned}
Q_{\ell} & =\sum_{d \in \mathbb{Z}^{*}} \sum_{n \in \Lambda_{0}} \mathbb{1}_{\Gamma}(n, n+d \ell) \mathbb{1}_{\Lambda_{0}}(n+d \ell) \Pi_{n} Q \Pi_{n+d \ell} . \\
Q_{0} & =\sum_{n \in \Lambda_{0}} \Pi_{n} Q \Pi_{n} .
\end{aligned}
$$

Using (5.12), we see that the last two conditions in (5.11) are satisfied. We still have to check that if $\Pi_{n} Q_{\ell} \Pi_{n^{\prime}} \neq 0$, then $n \in \Gamma_{\ell}, n^{\prime} \in \Gamma_{\ell}$. By (5.12), if $n^{\prime}=n+d \ell$,

$$
|d \ell|<\frac{1}{A}|n|^{\rho / 2},|F(n)-F(n+d \ell)| \leq \frac{1}{A}|n|^{\rho},
$$

so that writing $F(n+d \ell)=F(n)+F^{\prime}(n) \cdot(d \ell)+O\left(|d \ell|^{2}\right)$,

$$
|d|\left|F^{\prime}(n) \cdot \ell\right| \leq \frac{1}{A}|n|^{\rho}+C|d \ell|^{2}<\frac{1}{A}|n|^{\rho}\left(1+\frac{C}{A}\right) .
$$

Since $|d| \geq 1,|\ell| \geq 1$, we obtain for $A$ large enough relatively to $\frac{1}{\rho}$ that $n$ belongs to $\Gamma_{\ell}$. One obtains in the same way that $n^{\prime}=n+d \ell \in \Gamma_{\ell}$. This concludes the proof of the lemma.

Proof of Theorem 1.3: Let us check that condition (2.6) is satisfied when $\Lambda=-\Delta$. Actually, if $\Sigma \in \mathcal{L}_{\mathrm{ND}}^{\sigma}$, the equation $[Q,-\Delta]=\Sigma$ may be written, according to (5.3),

$$
\left(F\left(n^{\prime}\right)-F(n)\right) \Pi_{n} Q \Pi_{n^{\prime}}=\Pi_{n} \Sigma \Pi_{n^{\prime}}, n, n^{\prime} \in \Lambda_{0},
$$

and property (5.9) implies that we may find a solution $Q \in \mathcal{L}_{\mathrm{ND}}^{\sigma-\rho}$ of that equation. 
We are left with verifying conditions $(2.7)$ and (2.8). Let us define the operator $\tilde{\Delta}$. By (ii) of Lemma 5.2, for $\ell \neq \ell^{\prime}$ belonging to $\mathcal{P}$, the sets

$$
\Gamma_{\ell} \cap\left\{n ;|n|>B|\ell|^{\frac{2}{1-\rho}}\right\}, \Gamma_{\ell^{\prime}} \cap\left\{n ;|n|>B\left|\ell^{\prime}\right|^{\frac{2}{1-\rho}}\right\}
$$

are disjoint if $B$ is a large enough constant. We define $\tilde{\Delta}$ by the orthogonal sum

$$
\tilde{\Delta}=\sum_{\ell \in \mathcal{P}} \sum_{n \in \Lambda_{0}}\left(n \cdot \frac{\ell^{\perp}}{|\ell|}\right)^{2} \mathbb{1}_{\Gamma_{\ell}}(n) \mathbb{1}_{\left\{|n|>B|\ell|^{\frac{2}{-\rho}}\right\}} \Pi_{n}+\sum_{n \in \Omega}\left(1+|n|^{2}\right) \Pi_{n},
$$

where $\Omega$ is the complement in $\Lambda_{0}$ of

$$
\bigcup_{\ell \in \mathcal{P}}\left\{n ; n \in \Gamma_{\ell},|n|>B|\ell|^{\frac{2}{1-\rho}}\right\} .
$$

By (5.6), $\left|n \cdot \frac{\ell^{\perp}}{|\ell|}\right|^{2} \sim|n|^{2}$ when $n \in \Gamma_{\ell}$ so that, by orthogonality, condition (2.8) holds. By definition, the last two conditions $(2.7)$ hold as well. We just have to check that $[Q, \tilde{\Delta}] \in \mathcal{L}^{-\infty}$ for any $Q \in \mathcal{L}_{\mathrm{D}}^{\sigma}$. It is enough to verify that $\left[Q_{0}, \tilde{\Delta}\right]$ and $\sum_{\ell \in \mathcal{P}}\left[Q_{\ell}, \tilde{\Delta}\right]$ are in $\mathcal{L}^{-\infty}$ in decomposition (5.10). The case $Q_{0}$ is trivial. On the other hand, using (5.11)

$$
\begin{aligned}
\Pi_{n}\left[\tilde{\Delta}, Q_{\ell}\right] \Pi_{n^{\prime}}= & \left(n \cdot \frac{\ell^{\perp}}{|\ell|}\right)^{2} \mathbb{1}_{\Gamma_{\ell}}(n) \mathbb{1}_{\left\{|n|>B|\ell|^{\frac{2}{1-\rho}}\right\}} \Pi_{n} Q_{\ell} \Pi_{n^{\prime}} \\
& -\left(n^{\prime} \cdot \frac{\ell^{\perp}}{|\ell|}\right)^{2} \mathbb{1}_{\Gamma_{\ell}}\left(n^{\prime}\right) \mathbb{1}_{\left\{\left|n^{\prime}\right|>B|\ell|^{1-\rho}\right.} \Pi_{n} Q_{\ell} \Pi_{n^{\prime}} .
\end{aligned}
$$

By (5.11), if $\Pi_{n} Q_{\ell} \Pi_{n^{\prime}} \neq 0$, we have $n \in \Gamma_{\ell}, n^{\prime} \in \Gamma_{\ell}$ and $n-n^{\prime}$ collinear to $\ell$, so that

$$
\Pi_{n}\left[\tilde{\Delta}, Q_{\ell}\right] \Pi_{n^{\prime}}=\left(n \cdot \frac{\ell^{\perp}}{|\ell|}\right)^{2}\left[\mathbb{1}_{\left\{|n|>B|\ell|^{\frac{2}{1-\rho}}\right\}}-\mathbb{1}_{\left\{\left|n^{\prime}\right|>B|\ell|^{\frac{2}{1-\rho}}\right\}}\right] \Pi_{n} Q_{\ell} \Pi_{n^{\prime}} .
$$

If we use (5.4), together with the fact that by (5.11), $\left|n-n^{\prime}\right| \geq|\ell|$, and that $|\ell|^{\frac{2}{1-\rho}} \geq \frac{1}{B}|n|$ or $|\ell|^{\frac{2}{1-\rho}} \geq \frac{1}{B}\left|n^{\prime}\right|$, we obtain

$$
\left\|\sum_{\ell} \Pi_{n}\left[\tilde{\Delta}, Q_{\ell}\right] \Pi_{n^{\prime}}\right\|_{\mathcal{L}\left(L^{2}\right)} \leq C_{N}\left(1+|n|+\left|n^{\prime}\right|\right)^{-N}
$$

for any $N$. Since similar estimates hold for $\operatorname{Ad}^{k}\left(\partial_{t}\right) Q_{\ell}$, this shows that $\sum_{\ell}\left[\tilde{\Delta}, Q_{\ell}\right]$ is in $\mathcal{L}^{-\infty}$.

We have thus verified that assumptions (2.5) to (2.8) hold. We may therefore apply Theorem 2.1, whose conclusion implies Theorem 1.3.

\section{The linear $\mathrm{KdV}$ equation}

Let $W$ be as in the statement of Theorem 1.4, and let us check that we may apply Theorem 2.1 to equation (1.5). Set

$$
\Lambda=i \partial_{x}^{3}, V=\left(W i \partial_{x}+i \partial_{x} W\right)
$$


For $u \in L^{2}\left(\mathbb{S}^{1}\right)$ and $n \in \mathbb{Z}$, set

$$
\Pi_{n} u=\left\langle u, \frac{e^{i n x}}{\sqrt{2 \pi}}\right\rangle \frac{e^{i n x}}{\sqrt{2 \pi}}
$$

and define the space of operators $\mathcal{L}^{\sigma}$ by estimates (3.1). Then $\Lambda \in \mathcal{L}^{3}, V \in \mathcal{L}^{1}$ and we shall apply the results of section 2 with $m=3, \sigma_{0}=1, \rho=1$. We set

$$
\widetilde{\Pi}_{0}=\Pi_{0}, \widetilde{\Pi}_{n}=\Pi_{n}+\Pi_{-n}, n \in \mathbb{N}^{*},
$$

and define $\mathcal{L}_{\mathrm{D}}^{\sigma}$ and $\mathcal{L}_{\mathrm{ND}}^{\sigma}$ as those elements of $\mathcal{L}^{\sigma}$ satisfying respectively

$$
\begin{aligned}
& \widetilde{\Pi}_{n} Q \widetilde{\Pi}_{n^{\prime}}=0 \text { if } n \neq n^{\prime} \\
& \widetilde{\Pi}_{n} Q \widetilde{\Pi}_{n}=0 \text { for any } n \in \mathbb{N} .
\end{aligned}
$$

Then any element of $\mathcal{L}^{\sigma}$ may be decomposed under form (2.5). Let us check that (2.6) holds. The equation $\left[Q, i \partial_{x}^{3}\right]=\Sigma$ for $\Sigma \in \mathcal{L}_{\mathrm{ND}}^{\sigma}$ may be written

$$
\left(n^{\prime 3}-n^{3}\right) \Pi_{n} Q \Pi_{n^{\prime}}=\Pi_{n} \Sigma \Pi_{n^{\prime}}, n, n^{\prime} \in \mathbb{Z} .
$$

Since $\Sigma \in \mathcal{L}_{\mathrm{ND}}^{\sigma}$, we may assume $n \neq n^{\prime}$, which implies $\left|n^{\prime 3}-n^{3}\right| \geq c\left(1+|n|^{2}+\left|n^{\prime}\right|^{2}\right)$ for some $c>0$, so that $Q$ defined by (6.4) belongs to $\mathcal{L}_{\mathrm{ND}}^{\sigma-2}$, i.e. we get $(2.6)$ with $\sigma_{0}=\rho=1$.

Finally (2.7) is trivial, since we may take $\tilde{\Delta}=\Delta$. We may therefore apply Theorem 2.1. This concludes the proof of Theorem 1.4.

\section{References}

[1] J.-M. Barbaroux and A. Joye: Expectation values of observables in time-dependent quantum mechanics, J. Statist. Phys. 90 (1998), no 5-6, 1225-1249.

[2] A. Besse: Manifolds all of whose geodesics are closed, Ergebnisse der Mathematik und ihrer Grenzgebiete, 93. Springer-Verlag, Berlin-New York, 1978. ix+262 pp.

[3] J. Bourgain: Growth of Sobolev norms in linear Schrödinger equations with quasi-periodic potential, Comm. Math. Phys. 204 (1999), no. 1, 207-247.

[4] J. Bourgain: On growth of Sobolev norms in linear Schrödinger equations with smooth time dependent potential, J. Anal. Math. 77 (1999), 315-348.

[5] J. Bourgain: Global solutions of nonlinear Schrödinger equations, American Mathematical Society Colloquium Publications, 46. American Mathematical Society, Providence, RI, 1999. viii+182 pp.

[6] J. Bourgain: Green's function estimates for lattice Schrödinger operators and applications, Annals of Mathematics Studies, 158, Princeton University Press, 2005.

[7] Y. Colin de Verdière: Sur le spectre des opérateurs elliptiques à bicaractéristiques toutes périodiques, Comment. Math. Helv. 54 (1979), no. 3, 508-522. 
[8] Y. Colin de Verdière: Spectre conjoint d'opérateurs pseudo-différentiels qui commutent I. Le cas non intégrable, Duke Math. J. 46 (1979), no. 1, 169-182.

[9] Y. Colin de Verdière: Spectre conjoint d'opérateurs pseudo-différentiels qui commutent II. Le cas intégrable, Math. Z. 171 (1980), no. 1, 51-73.

[10] J. Duistermaat and V. Guillemin: The spectrum of positive elliptic operators and periodic bicharacteristics, Invent. Math. 29 (1975), no. 1, 39-79.

[11] H. Eliasson and S. Kuksin: On reducibility of Schrödinger equations with quasiperiodic potentials, Comm. Math. Phys. 286 (2009), no. 1, 125-135.

[12] V. Guillemin: Lectures on spectral theory of elliptic operators, Duke Math. J. 44 (1977), no. $3,485-517$.

[13] W.-M. Wang: Logarithmic bounds on Sobolev norms for time dependent linear Schrödinger equations, Comm. Partial Differential Equations 33 (2008), no. 10-12, 2164-2179.

[14] W.-M. Wang: Bounded Sobolev norms for linear Schrödinger equations under resonant perturbations, J. Funct. Anal. 254 (2008), no. 11, 2926-2946.

[15] A. Weinstein: Asymptotics of eigenvalue clusters for the Laplacian plus a potential, Duke Math. J. 44 (1977), no. 4, 883-892. 